FORMATION Formation emploi

Revue française de sciences sociales

100 | octobre-décembre 2007

De la formation professionnelle en Suisse

\title{
Le système suisse de formation face aux mutations du marché du travail
}

Yves Flückiger

\section{(2) OpenEdition}

1 Journals

Édition électronique

URL : http://journals.openedition.org/formationemploi/1251

DOI : 10.4000/formationemploi.1251

ISSN : 2107-0946

Éditeur

La Documentation française

Édition imprimée

Date de publication : 1 octobre 2007

Pagination : 5-14

ISSN : 0759-6340

Référence électronique

Yves Flückiger, «Le système suisse de formation face aux mutations du marché du travail »,

Formation emploi [En ligne], 100 | octobre-décembre 2007, mis en ligne le 23 septembre 2008, consulté le 30 octobre 2020. URL : http://journals.openedition.org/formationemploi/1251 ; DOI : https:// doi.org/10.4000/formationemploi.1251

(c) Tous droits réservés 


\title{
INTRODUCTION
}

\section{Le système suisse de formation face aux mutations du marché du travail}

\author{
Par Yves Flückiger ${ }^{\star}$
}

Le marché suisse du travail a connu de profondes mutations au cours des dernières décennies. Elles se sont traduites, en particulier, par une hausse marquée du chômage, notamment lors des années 90 , et par des difficultés croissantes de transition des jeunes vers l'emploi, notamment en fin de formation. Elles se sont aussi manifestées par une flexibilité accrue imposée par les entreprises à des employés qui changent de plus en plus fréquemment d'employeurs ou de professions.

Contrairement à ce que de nombreux économistes prétendent encore, ces changements ne sont pas seulement de simples accidents conjoncturels, mais ils représentent des phénomènes de long terme. Or, il est aujourd'hui incontesté que la formation et, de manière plus générale, le « capital humain » accumulé tout au cours de l'existence, constitue un facteur particulièrement important pour prévenir le chômage ou pour faciliter la réinsertion professionnelle des individus qui auraient perdu leur emploi. Pourtant, à considérer le comportement des personnes actives, les stratégies développées par bon nombre d'entreprises ou la politique de formation suivie par la Suisse, il faut admettre que ce constat, pourtant très largement partagé, n'est pas encore suivi d'effets, loin s'en faut.

Par les thèmes qu'il aborde, ce dossier de la revue «Formation Emploi: revue française de sciences sociales 》, consacré à la Suisse, est particulièrement intéressant car il analyse, sous différentes facettes, les défis que le système de formation devra affronter.

\section{LES SIGNES DES MUTATIONS DE L'EMPLOI}

Une analyse attentive de l'évolution du marché du travail en Suisse permet de déceler les signes des mutations en cours dans notre pays. Malheureusement, à l'exception de l'Enquête suisse sur la population active (ESPA), réalisée par l'Office fédéral de la statistique sur une base annuelle, il faut reconnaître que les données statistiques nécessaires pour appréhender la réalité de ces évolutions à moyen et long terme font encore cruellement défaut. Une telle lacune

* Yves Flückiger est professeur au département d'économie politique, université de Genève, directeur de l'Observatoire universitaire de l'emploi et de la "Leading House » en économie de l'éducation de l'université de Genève. Il est l'auteur notamment de "Problèmes de l'État social : causes, fondements et perspectives", publié en 2007, en collaboration avec L. Gärtner, et de "Les différences régionales en matière de chômage: une approche renouvelée", publié par La vie économique en 2007, en collaboration avec $P$. Kempeneers et $S$. Bazen. 
ne favorise évidemment pas la prise de conscience, pourtant indispensable, des autorités politiques.

L'analyse de la relation entre l'emploi et le Produit intérieur brut (PIB) est particulièrement révélatrice des changements actuels. Durant les années 1974 à 1976, en pleine période de récession économique, chaque diminution de $1 \%$ du PIB, en termes réels, s'est traduite par une contraction de $0,9 \%$ seulement du nombre d'offres d'emplois. En comparaison, au cours de la période de 1991 à 1995, l'économie suisse a enregistré une diminution de près de $6 \%$ de l'emploi alors même que le volume du PIB stagnait au niveau qui était le sien en 1991. La même relation a été observée au cours de la dernière récession économique qui a affecté l'économie helvétique, au début des années 2000. Il semble donc que les périodes de récession économique entraînent, en Suisse comme dans tous les pays de l'OCDE (Organisation de coopération et de développement économiques), une destruction plus importante de postes de travail que par le passé.

$\mathrm{Au}$ lieu de conserver leurs effectifs intacts dans l'espoir d'un redémarrage rapide de l'activité, comme elles l'avaient fait au milieu des années 70, les entreprises suisses ont été amenées à réduire massivement leur volume d'emploi au début des années 90. Leur attitude s'explique en premier lieu par le fait que bon nombre de sociétés sont engagées dans un processus de restructuration de leurs activités qui implique le recours à des techniques de production plus mécanisées et à une main-d'œuvre plus qualifiée. Dès lors, il n'est pas surprenant de constater que la productivité du travail a augmenté au cours des dernières années, en particulier lors des années caractérisées par une hausse du chômage.

Ce changement d'attitude prouve que les entreprises investissent aujourd'hui moins dans la formation continue de leur main-d'œuvre ( $C f$. Office fédéral de la statistique, 2007) contrairement aux années 70 , où elles ont cherché à conserver leur personnel pour lequel elles avaient consenti d'importants efforts de formation. En effet, si les employeurs diminuent leurs investissements en capital humain, une politique de thésaurisation du personnel, concrétisée par des relations contractuelles de travail à long terme, ne se justifie plus alors qu'elle était indispensable dans le passé pour amortir les dépenses de formation consenties par les entreprises. Une autre preuve de ce changement provient du fait que les employeurs ont eu moins recours au chômage partiel lors des dernières récessions économiques qu'elles ne l'ont fait vingt ou trente ans auparavant.

Le deuxième indicateur des changements en cours concerne la flexibilité que les entreprises cherchent à imposer au marché du travail. Si cette stratégie peut prendre différentes formes, en termes de temps de travail notamment, elle se manifeste en particulier par une flexibilité fonctionnelle qui implique une polyvalence des travailleurs et une diversification de leurs tâches.

La figure 1 illustre cette exigence accrue par le biais de la mobilité professionnelle des personnes actives, enregistrée lors des trois derniers recensements fédéraux de la population ${ }^{1}$. Elle indique que la part des personnes actives en Suisse qui ont changé de profession par rapport à celle qu'elles avaient apprise n'a cessé de croître au cours des trente dernières années, et ceci quels que soient la classe d'âge, le secteur d'activité ou la nationalité. Cette observation donne un certain crédit à l'adage selon lequel on n'apprend plus aujourd'hui une profession pour toute sa vie mais qu'il faut se préparer à devoir changer de profession, et ceci sans doute plusieurs fois. Dans ce contexte, il est évident que la formation de base doit s'adapter à ces exigences nouvelles en transmettant les compétences nécessaires à la mobilité future sur le marché du travail. Mais la formation continue joue sans doute un rôle encore plus crucial pour faciliter les changements professionnels en maintenant à jour les connaissances de base acquises par les individus.

Finalement, depuis le début des années 90, on assiste à un recours de plus en plus systématique à la flexibilité chronologique. Cette évolution est particulièrement perceptible au niveau de l'accroissement du nombre d'indépendants et du nombre de personnes occupées à temps partiel. Or, on sait que les personnes occupées à temps partiel, les femmes en particulier, accèdent moins fréquemment à des cours de formation continue que les employés à pleintemps (Cf. Office fédéral de la statistique, 2007).

\footnotetext{
${ }^{1}$ Dans le cadre de ces recensements exhaustifs, on enregistre la profession apprise par les personnes interrogées ainsi que leur dernière profession exercée en utilisant la même grille de lecture basée sur une classification en 86 groupes différents.
} 
Figure 1

Proportion de personnes actives exerçant une profession différente de celle qu'elles ont apprise, RFP (recensements fédéraux de la population), 1980 - 2000

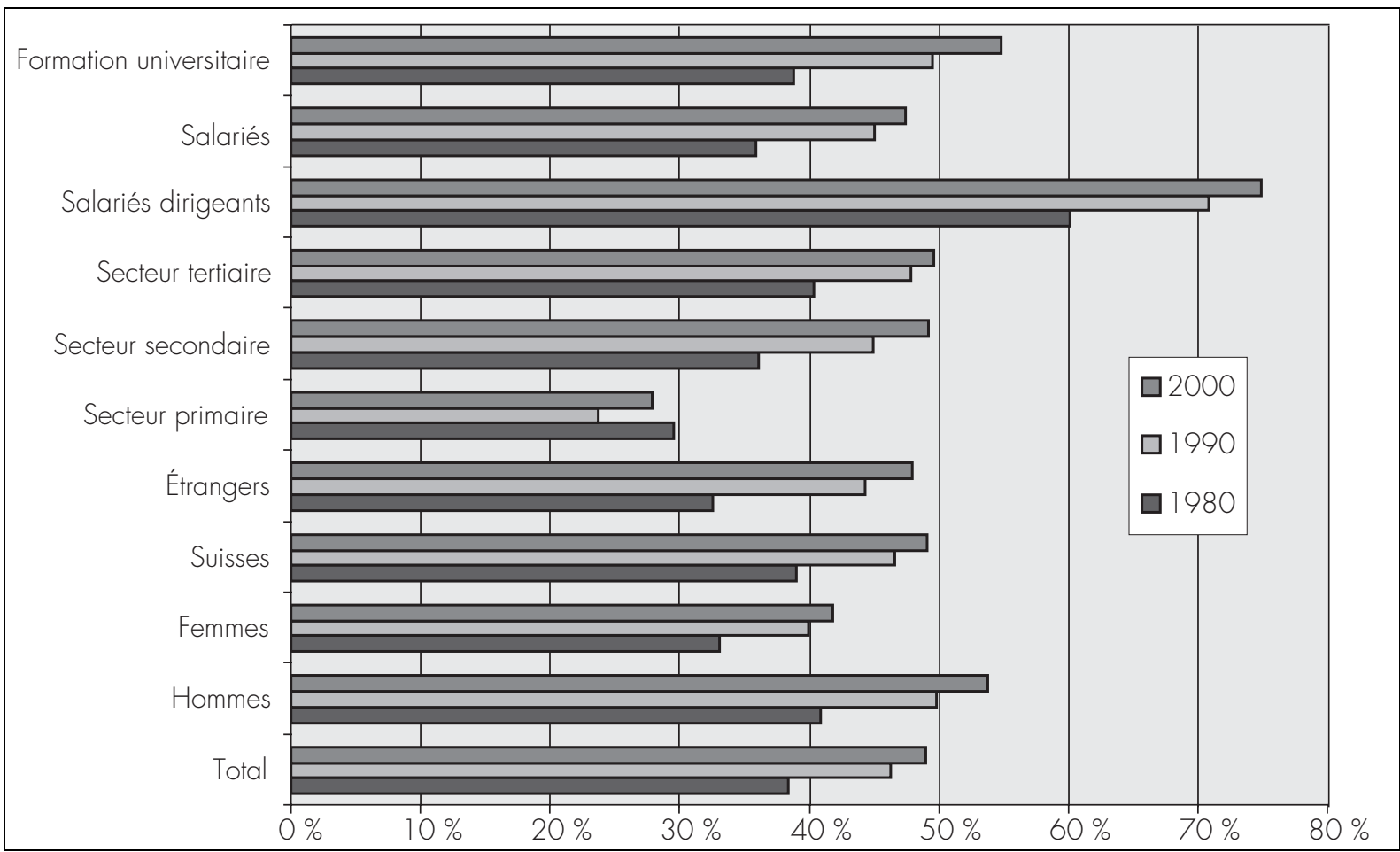

Source : OFS, RFP, 1980-2000.

\section{Figure 2}

Proportion de personnes actives travaillant moins de $90 \%$ du temps de travail normal de leurs entreprises, ESPA (Enquête suisse sur la population active), 1991-2005

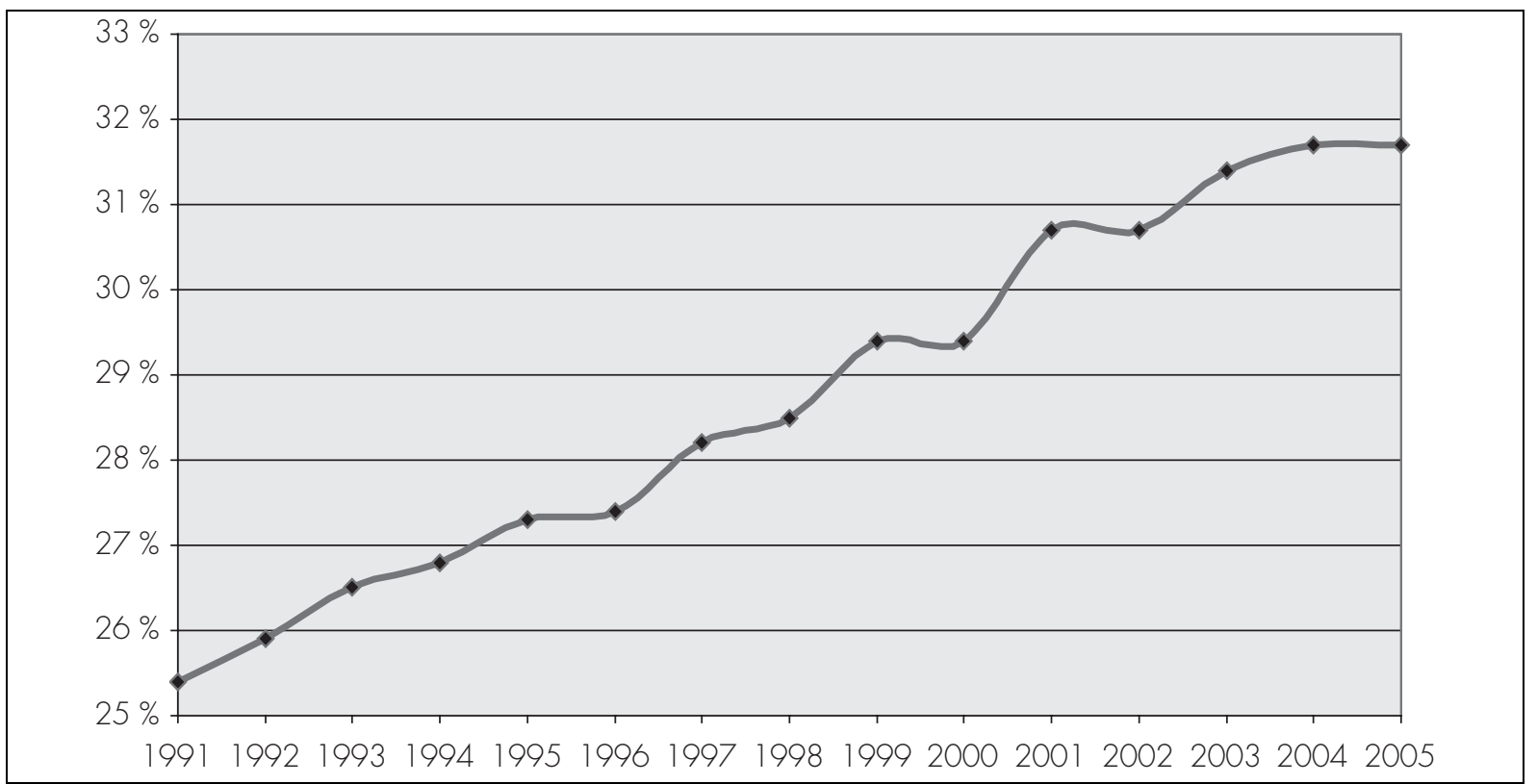

Source : OFS, ESPA, 1991-2005. 
Le développement du nombre des indépendants traduit le même phénomène. Il reflète la politique «d'outsourcing» (d'externalisation) pratiquée par un nombre croissant d'employeurs qui cherchent à externaliser des tâches qu'ils confiaient auparavant à des personnes salariées intégrées à l'entreprise. Même si ce phénomène reste encore modeste, le revirement de tendance à long terme est significatif. En effet, historiquement, on observe que le pourcentage d'indépendants a constamment diminué depuis le début du siècle. Cette tendance séculaire s'est renversée aux États-Unis tout d'abord, dès le début des années 70 , puis dans les pays de l'Union européenne, pour toucher plus récemment la Suisse. D'après les données des deux derniers recensements fédéraux de la population, le taux d'indépendants est passé de $9,7 \%$ en 1980 à $10,6 \%$ en 1990 . La récession économique du début des années 90 n'a fait que renforcer davantage cette tendance, puisque bon nombre de chômeurs trouvent dans le statut d'indépendant une voie de sortie, souvent précaire, vers un emploi rémunéré. En 2006, le pourcentage d'indépendants atteint $13,6 \%$. Si on y ajoute les collaborateurs d'entreprises familiales, ce taux s'élève à $17,1 \%$. Or, force est de constater que le perfectionnement professionnel vise avant tout les personnes salariées et que les indépendants n'ont souvent ni le temps ni les moyens d'assurer la mise à jour permanente de leurs compétences.

\section{LES CONSÉQUENCES POUR LE SYSTÈME DE FORMATION}

Les conséquences du développement des nouvelles formes de travail se font particulièrement sentir dans le domaine de la formation continue. Comme nous avons déjà eu l'occasion de le dire, les entreprises helvétiques tendent à réduire, à l'heure actuelle, leurs efforts dans ce domaine. Dans le même temps, il faut admettre que l'attitude de la population suisse face à la formation permanente ne correspond pas à celle que l'on pourrait attendre dans cette période de mutations économiques. Les quelques enquêtes réalisées en Suisse sur le thème de la formation continue démontrent qu'une fraction encore relativement faible de la population participe à une activité de perfectionnement. Ainsi, selon une enquête menée par l'Office fédéral de la statistique (OFS, 2007), cette proportion atteint $31 \%$ de la population adulte âgée de 20 à 74 ans en 2006 et est en diminution depuis dix ans puisqu'elle s'élevait encore à $37 \%$ en 1996.

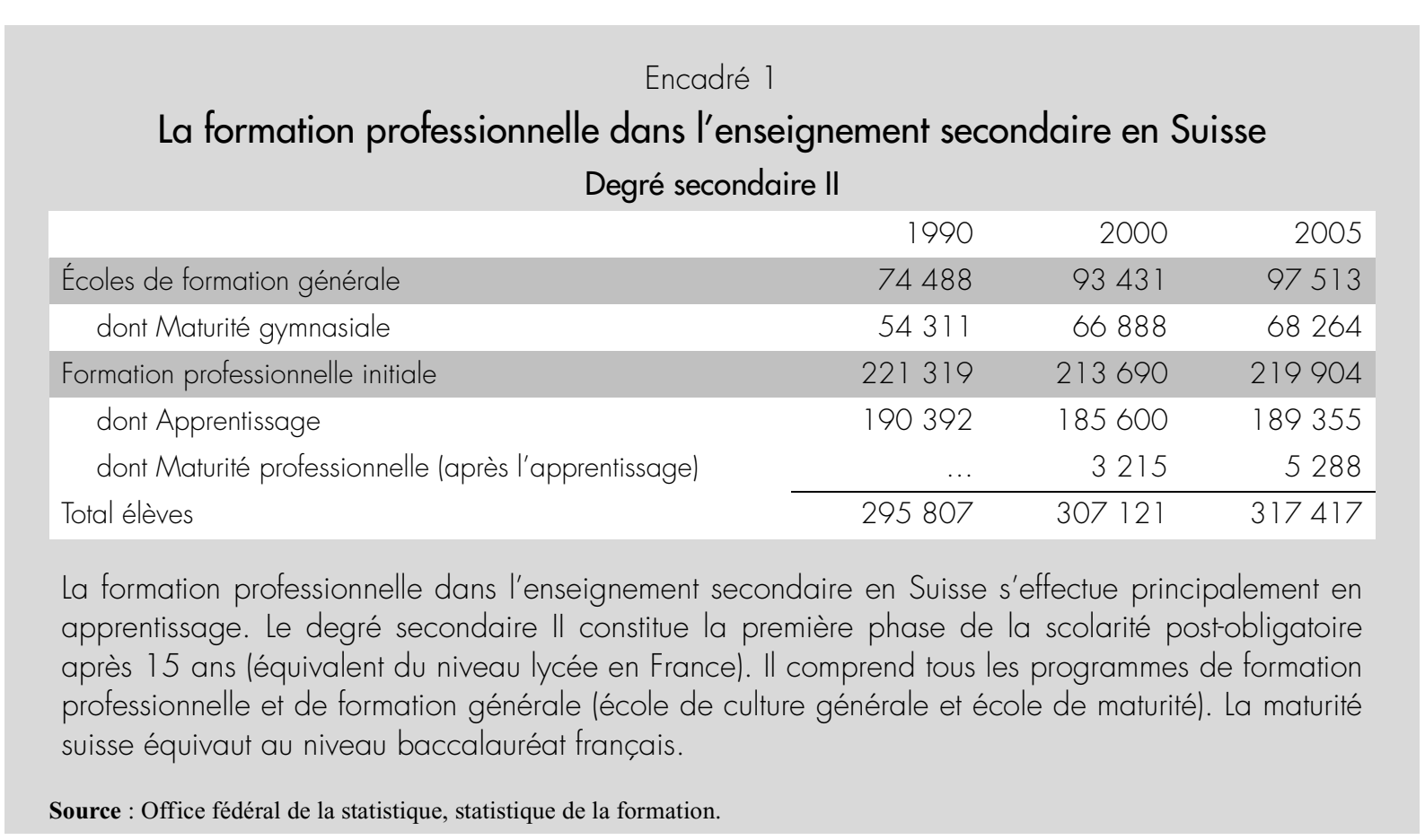


Il apparaît donc que le marché de la formation continue en Suisse est défaillant (Booth et Snower, 1996). Cette défaillance provient notamment du fait que, dans une période de flexibilisation croissante du marché du travail et de mobilité accrue des travailleurs, les employeurs ont tendance à moins investir dans la formation continue de leur propre personnel pour profiter des efforts consentis par les autres entreprises. Ce déficit peut être comblé par des interventions de l'État mais celles-ci doivent être réfléchies pour éviter qu'une lacune du marché ne soit pas tout simplement remplacée par une autre défaillance émanant cette fois de l'État.

La question cruciale de la formation continue est traitée de manière à la fois exhaustive et originale par trois articles de ce dossier consacré à la Suisse. Le premier, celui de Siegfried Hanhart, porte précisément sur le comportement des entreprises suisses en matière de formation professionnelle continue (FPC). Il rappelle en premier lieu qu'en Suisse, cette formation relève d'abord de la responsabilité des individus et des entreprises, la Confédération et les cantons agissant subsidiairement. Dans ce contexte, il est évidemment particulièrement important d'appré- hender le comportement des entreprises à l'égard de la formation permanente de leurs collaborateurs. Or, comme nous l'avons mentionné auparavant, il n'existe quasiment pas de données statistiques à ce propos et encore moins d'enquêtes. De ce point de vue, l'article de Siegfried Hanhart est particulièrement éclairant puisqu'il s'appuie sur une vaste enquête menée entre 2000 et 2003 , dans le cadre d'un programme national de recherche, auprès de 1750 entreprises privées et publiques en Suisse questionnées sur leurs dépenses de FPC. Dans cet article, l'auteur présente et discute les principaux résultats de cette enquête en ce qui concerne le profil des participants et les activités de FPC soutenues par les entreprises, les dépenses consenties par les employeurs, les modalités d'évaluation des effets de la FPC mises en œuvre dans les firmes, et les raisons évoquées par les employeurs pour soutenir ou pas la formation continue de leurs collaborateurs. Ces résultats sont également comparés avec des données européennes. Il en ressort que les entreprises suisses investissent relativement peu et de manière très sélective dans la formation continue de leur personnel.

\section{Les 26 cantons suisses}

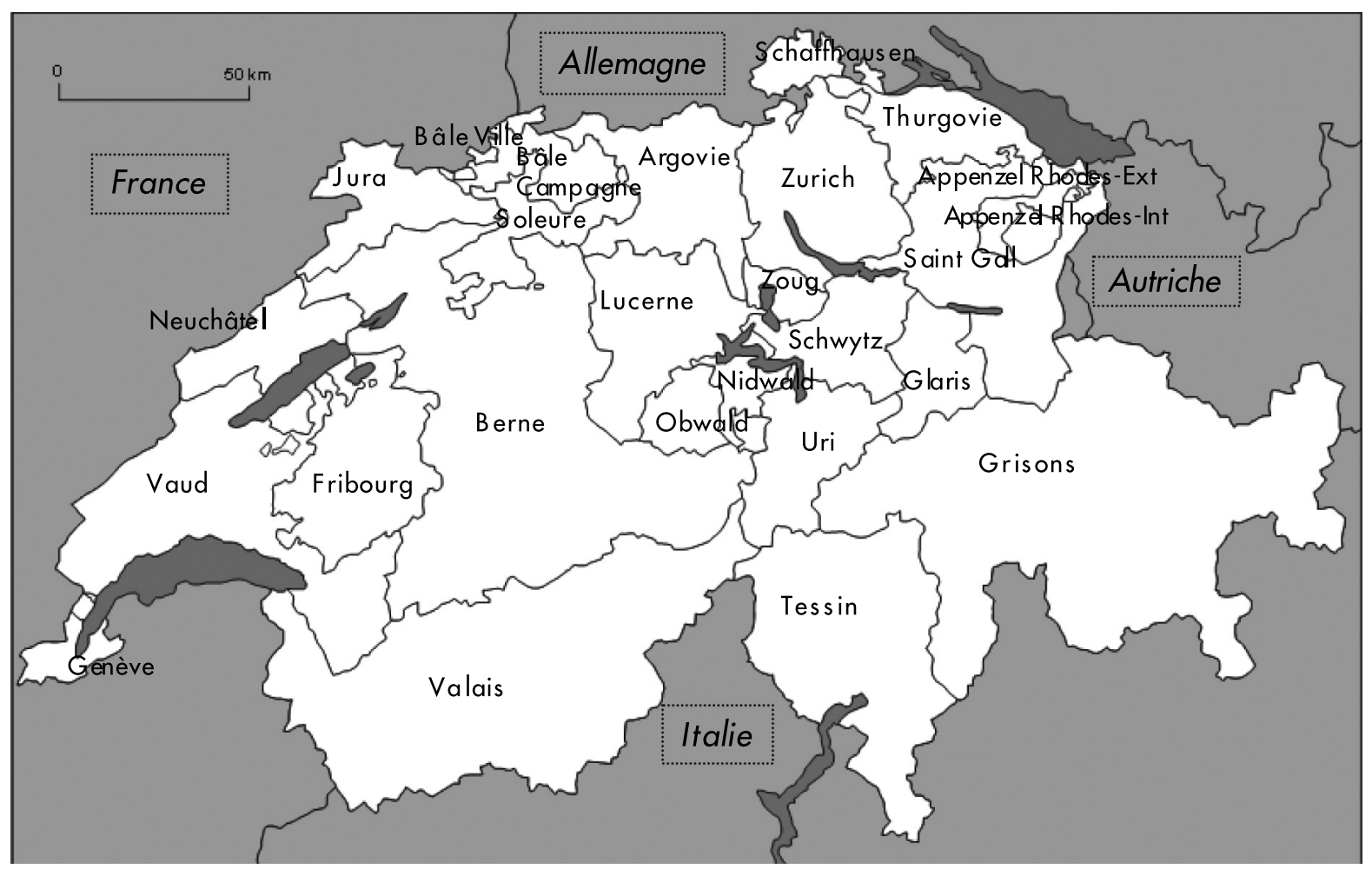


Si l'attitude des entreprises est importante à appréhender, celle des employés est tout aussi intéressante à comprendre afin d'élaborer les politiques publiques adéquates. C'est en particulier l'objectif d'Yves Emery dans son article consacré au comportement des employés des services publics occupés dans un environnement managérialisé. L'auteur y met en évidence les différentes stratégies que les agents publics suisses mettent en œuvre, au sein d'organisations soumises à une managérialisation croissante, pour atteindre leurs propres objectifs de formation. Sur la base de ces analyses, l'auteur esquisse certains enjeux plus fondamentaux relatifs à l'avenir de la formation continue dans les services publics suisses, enjeux qui sont d'ailleurs largement transposables au sein d'autres pays de l'OCDE.

Toutes les études réalisées en Suisse sur le thème du chômage (notamment Flückiger et al., 2007) démontrent que le risque de chômage mais plus encore sa durée et les chances de retrouver un travail sont largement déterminés par le niveau de formation initiale atteint par chaque personne et par la formation continue dont elles ont pu bénéficier au cours de leur vie. Quoi qu'il en soit, celle-ci est nettement plus efficace lorsqu'elle est suivie en cours d'emploi plutôt qu'en période de chômage. De ce point de vue, l'article d'Olivier Giraud est particulièrement intéressant puisqu'il porte sur une analyse des politiques d'activation des chômeurs suisses et de leur mise en œuvre dans un contexte fédéraliste. Celui-ci laisse une grande autonomie de mise en œuvre des mesures prévues par la loi fédérale en matière de chômage. Cette autonomie peut être source de plus grande efficacité en permettant à chaque canton de s'organiser de la manière la plus adéquate possible par rapport à sa situation particulière et d'adopter les mesures qui lui paraissent les plus appropriées. Mais elle est aussi, comme le montre fort bien l'auteur, à l'origine de divergences d'interprétation qui apparaissent clairement dans les discours et les usages des politiques de formation développées en Suisse pour les chômeurs. L'auteur postule que ces divergences proviennent principalement $\mathrm{du}$ caractère polysémique de la notion d'activation attachée aux politiques de la formation et de l'emploi. De ce point de vue, il apparaît que la décentralisation d'un système politique suppose l'existence d'arènes de pouvoir autonomes les unes par rapport aux autres, qui sont autant d'espaces potentiels permettant le développement de tels conflits d'interprétation, mais aussi de formes contrastées de mise en œuvre des politiques publiques.

Dans le contexte de mutations de l'emploi qui caractérise le marché suisse du travail, la formation professionnelle duale, qui a fait très longtemps la force de la Suisse, est soumise aujourd'hui à des tensions de plus en plus fortes, liées notamment aux adaptations qu'il faudrait apporter à ce système dual pour lui permettre de mieux répondre aux restructurations économiques et à l'augmentation des exigences qualitatives en matière de formation. De ce point de vue, l'article de Laurence Marti est d'un apport précieux quant à la situation et aux évolutions en cours, puisqu'il s'appuie sur les études menées par l'auteur dans trois branches d'activités différentes: la restauration, l'horlogerie et la fabrication de machines.

L'article de Laurence Marti est complété par l'analyse proposée par Jonas Masdonati, Nadia Lamamra, Benôt Gay-des-Combes et Jacqueline De Puy qui questionnent le rôle de la formation professionnelle duale dans la construction identitaire des apprenants. Le matériel utilisé pour ce faire provient de deux recherches en cours à l'Institut fédéral des hautes études en formation professionnelle, qui portent sur les apprenants en première année de formation. Les premiers résultats indiquent que ce système de formation présente aussi bien des forces que des faiblesses quant à sa contribution au développement identitaire des apprenants. Cet état des lieux débouche sur des perspectives concrètes visant à renforcer les atouts et à remédier aux inconvénients de la formation professionnelle duale, tels que l'aménagement de la transition entre école et formation professionnelle ou la valorisation du rôle des formateurs en entreprise.

Si la Suisse est caractérisée par un système de formation particulier qui en rend l'analyse intéressante par sa diversité cantonale et le développement de son système dual, elle se trouve aussi au milieu de l'Europe, entourée de pays avec lesquels elle a signé un accord bilatéral prévoyant la libre circulation de la main-d'œuvre. Ce régime, dont les effets ont 
commencé à se faire ressentir à partir de 2004, a suscité beaucoup de craintes parmi la population, compte tenu des conséquences supposées de cette libre circulation sur les salaires des personnes actives sur le marché du travail suisse. Il existe pourtant relativement peu d'études sur les conséquences de cette libre circulation. Même si l'article de Romain Felli, Gaële Goastellec et Jean-Philippe Leresche porte sur une facette limitée de la mobilité des personnes, il constitue néanmoins un cas d'étude fort appréciable. En l'occurrence, les auteurs examinent les interrelations ou les interdépendances entre les marchés de l'emploi académique suisse et français. Ils tentent ainsi de déterminer si le système d'enseignement supérieur et de recherche français constitue un marché pour les universitaires suisses et réciproquement. Consacrée à la mise en perspective des grandes tendances internationales des marchés de l'emploi académique, une première partie de l'article éclaire la comparaison des situations suisse et française. Ensuite, sur la base de résultats empiriques, les interrelations entre ces deux marchés sont analysées quantitativement. Puis les auteurs examinent les instruments de coopération scientifique mis en place entre les deux pays. Enfin, différents facteurs sont mobilisés (effet taille, logiques disciplinaires, attractivité, etc.) pour expliquer les convergences et différences d'imbrication des marchés du travail académique suisse et français afin d'interroger la possible intégration d'un marché franco-suisse des universitaires dans un contexte européen.

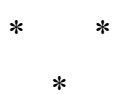

Les nouvelles formes de travail constituent sans doute une source d'emplois bienvenue pour l'économie suisse. Elles représentent en même temps un défi pour la politique de formation, notamment dans le domaine de la formation continue. Il faut en prendre conscience pour élaborer les réformes qui permettront à la Suisse d'affronter ces mutations en évitant les conséquences néfastes qui pourraient les accompagner.

Tandis que la formation de base en Suisse, enseignement supérieur inclus, se caractérise par une offre essentiellement assurée et financée par l'État, c'est exactement le contraire dans le domaine du perfec- tionnement et de la formation continue. La seule exception, de taille, concerne les programmes et cours de perfectionnement destinés aux chômeurs dans le cadre de la politique active de l'emploi. Si cette politique est adaptée aux besoins actuels, elle a néanmoins ses limites. Pour pouvoir bénéficier de ces mesures, il faut en effet être au chômage. Or, dans ce cas, l'autorité chargée de l'application des mesures actives se trouve placée devant une tâche difficile, celle de devoir compenser, en peu de temps, les lacunes en matière de formation qui se sont accumulées tout au long de la carrière professionnelle de la personne concernée par ces mesures. Dans la plupart des cas, une intervention plus précoce aurait été plus efficace et moins coûteuse. Cette politique est d'autant plus délicate que le pourcentage de personnes qui, en Suisse, ne disposent pas d'une formation au-delà de la scolarité obligatoire est encore beaucoup trop élevé, puisqu'il atteint plus de $35 \%$. Il existe donc à l'heure actuelle une insuffisance chronique du côté de la formation de base qui ne peut pas être résolue simplement par le biais de la politique de formation continue.

Pour résoudre cela, différentes solutions sont possibles. Tout d'abord, l'État pourrait envisager $\mathrm{d}$ 'instaurer une aide publique directe ou indirecte (par le biais, par exemple, d'une réduction des cotisations chômage) destinée aux entreprises qui initient des cycles de formation continue pour leur personnel ou qui instituent, dans le cadre de leurs contrats de travail, des systèmes dans lesquels leurs employés peuvent accumuler des crédits de formation susceptibles d'être valorisés après une période donnée passée au sein du même établissement. Un tel aménagement du temps de travail, sur une durée de vie plus longue, profiterait à la collectivité et aux travailleurs (grâce à une diminution de la probabilité de chômage) tout en permettant aux entreprises d'augmenter la productivité du personnel en place. Les employeurs peuvent ainsi fidéliser leurs employés grâce au système de crédit, tout en rentabilisant mieux leurs investissements de formation.

Une autre piste intéressante est celle du chèque annuel de formation mis en place, en Suisse, par le canton de Genève, en 2001. Ce chèque a une valeur annuelle de Fr. 750.- (équivalent à un peu plus de 440 euros) et il peut être attribué trois années de suite. Il permet de 
financer tout ou partie d'un cours utile sur le plan professionnel. La durée du cours choisi doit être de 40 heures minimum. Toute personne majeure domiciliée et contribuable dans le canton de Genève depuis une année au moins, au moment où la demande de formation est formulée, est éligible si son revenu correspond à certaines normes; ainsi la limite du revenu brut annuel du demandeur a été fixée à Fr. 88340 .-, équivalent à 52000 euros $^{2}$ pour une personne célibataire, séparée ou divorcée, montant qui s'accroît en fonction du nombre d'enfants à charge.

Une troisième solution consisterait à adopter ce que l'on pourrait appeler le «modèle danois ». Confronté à un taux de chômage deux fois plus important qu'en Suisse, le gouvernement danois a décidé de se doter d'un nouvel instrument d'intervention visant à compléter la panoplie des mesures actives en vigueur dans ce pays. En effet, depuis 1994, la population danoise a la possibilité de bénéficier, sous certaines conditions, d'un congé de formation qui vise à encourager les personnes actives à se former et à se perfectionner tout en favorisant la réintégration des chômeurs dans la vie active en leur permettant de remplacer temporairement les personnes en formation. Sans entrer dans les détails, précisons que pour bénéficier de ce système il faut avoir 25 ans au minimum, et avoir exercé une activité professionnelle pendant trois ans minimum au cours des cinq dernières années. La durée du congé va de une à cinquante deux semaines durant lesquelles la personne en formation reçoit l'équivalent de l'indemnité journalière à laquelle elle aurait eu droit en cas de chômage. Le congé de formation requiert l'assentiment de l'employeur mais il est ouvert également aux chômeurs et aux indépendants et l'employeur n'est pas tenu d'embaucher un(e) remplaçant(e).

$\mathrm{Ce}$ système présente plusieurs avantages. Tout d'abord, les personnes actives sont encouragées à se perfectionner sans attendre pour cela de se retrouver au chômage. Le perfectionnement des actifs devrait donc être plus efficace en évitant de trop longues périodes sans formation. D'autre part, pour les

${ }^{2}$ Ce montant équivaut à 1,2 fois le salaire médian obtenu par les personnes occupées dans le secteur privé de l'économie genevoise. À ce propos, il convient de rappeler qu'il n'existe pas, en Suisse, de salaire minimum pour l'ensemble de l'économie. chômeurs qui remplacent les actifs en formation, on évite les conséquences d'un chômage qui s'éternise tout en augmentant leurs chances de réinsertion rapide grâce aux contacts qu'ils nouent au sein de l'entreprise. De même, la participation de leurs employés à une formation continue devrait permettre aux employeurs de bénéficier de gains futurs de productivité tout en réduisant leurs coûts de formation et d'embauche. Par ce biais, ils ont en effet la possibilité de tester des collaborateurs potentiels. Finalement, l'État profite d'une amélioration de la compétitivité de l'économie. Dans le même temps, les chances de réinsertion professionnelle des chômeurs s'améliorent et la probabilité de chômage pour les actifs occupés diminue.

Malgré ces avantages indéniables, les premières évaluations réalisées sur cette expérience novatrice montrent que tout n'est pas encore parfait. Ainsi, la majorité des personnes qui ont participé au système étaient déjà au chômage. Seules $40 \%$ d'entre elles exerçaient une activité. En ce qui concerne la durée des formations, les résultats sont plus encourageants puisque les personnes actives occupées ont choisi des formations d'une durée moyenne de quatre mois, alors que les chômeurs ont opté pour des formations encore plus longues (sept mois en moyenne). De leur côté, les employeurs ont privilégié le recours au congé de formation pour le personnel le plus qualifié qu'ils souhaitaient conserver au sein de leur entreprise. Dès lors, les employeurs ont cherché des remplaçants relativement qualifiés qu'ils ont rarement trouvé parmi les personnes au chômage. L'effet espéré pour les chômeurs est donc resté faible.

En résumé, on peut dire que si cette expérience danoise présente des éléments dignes d'intérêt, elle comporte aussi ses limites. Ainsi, il semble bien que ce système ne permette pas de combler le fossé existant entre les personnes qualifiées et celles qui le sont moins. $\mathrm{Au}$ contraire, il tendrait même à le creuser. De surcroît, les chômeurs qui ont participé à des cours de formation sont ceux qui possédaient les meilleures chances de s'en sortir. Il est possible dès lors que le congé de formation ait contribué à prolonger la durée de chômage. Finalement, l'État risque bien de financer les efforts de perfectionnement des entreprises qui auraient de toute façon eu recours à des cours de formation en les prenant en 
charge elles-mêmes. On peut donc craindre que ce système ait tout simplement incité les employeurs à « externaliser » les coûts de la formation continue qu'ils supportaient auparavant.

Pour éviter ces défaillances, il faudrait axer cette politique sur les personnes actives peu qualifiées, en établissant par exemple des conditions d'admission plus strictes. On pourrait ainsi envisager de fixer des limites supérieures de formation de base au-delà desquelles les personnes ne seraient plus éligibles. Alternativement, on pourrait préciser que seules les personnes qui n'ont plus bénéficié de mesures de perfectionnement professionnel au cours des cinq dernières années par exemple peuvent obtenir un congé de formation. Dans le même état d'esprit, il conviendrait d'obliger l'entreprise à remplacer les employés en formation par un chômeur. Finalement, il s'agirait de fixer une durée maximale de formation mais aussi une durée minimale pour éviter que l'État ne finance des formations de très courte durée dont l'efficacité reste limitée. Ces restrictions réduiraient immanquablement le nombre de personnes susceptibles d'obtenir un congé de formation; cependant, elles sont susceptibles d'améliorer l'efficacité globale $\mathrm{du}$ système tout en minimisant les risques de défaillance observés au Danemark. Cela ne pourrait que le rendre plus efficace, moins coûteux et plus acceptable pour l'ensemble de la population.

\section{Bibliographie}

Booth A. L. et Snower D. J. (1996), Acquiring skills market failures, theirs symptoms and policy responses, Cambridge University Press.

Burdett K. et Smith E. (1996), "Education and matching externalities?", in Booth and Snower, pp. 63-80.

Flückiger Y. (1996a), «Les nouvelles pistes pour le financement des assurances sociales », Aspects de la Sécurité Sociale, Bulletin de la FEAS, 2-3/1996, pp. 47-62.

Flückiger Y. (1996b), La situation des chômeurs en fin de droit à Genève, Rapport d'activité 1995 de l'OCE, Département de l'économie publique, Genève, pp. 34-47.

Flückiger Y. et Morales D. (1994), « Analyse des causes de l'augmentation actuelle du chômage en Suisse et à Genève ", Série de publications du LEA, $\mathrm{n}^{\circ} 5$, université de Genève.
Flückiger Y. (2002), "Le chômage en Suisse : causes, évolutions et efficacité des mesures actives ", Aspects de la sécurité sociale, 4, pp. 11-21.

Flückiger Y., Kempeneers P. et Bazen S. (2007), «Les différences régionales en matière de chômage : une approche renouvelée », La vie économique, 7/8.

Ferro Luzzi G., Flückiger Y., Ramirez J. et Vassiliev A. (2006), "Unemployment and Employment Offices' Efficiency: What can be done?", Socio-Economic Planning Sciences, 40, pp. 169-186.

Gärtner L. et Flückiger Y. (2006), Problèmes de l'État social: causes, fondements et perspectives, Verlag Rüegger, Zürich.

Office fédéral de la statistique (1996), «Communiqué de presse : statistique du volume de travail $1994 »$, Sake-News, n 1/96, Berne.

Office fédéral de la statistique (1996), La société de formation - un mythe?, BFS aktuell, Berne. 
Office fédéral de la statistique (2007), Participation à la formation continue en Suisse : premiers résultats du module "Formation continue" de l'enquête suisse sur la population active de 2006, OFS, Neuchâtel.
Wolter S. C. et Knucherl B. (1997), "Formation pour les personnes actives, travail pour les chômeurs", $L a$ Vie économique, 5/97, pp. 22-28.

Stevens M. (1996), "Transferable training and poaching extrenalities", in Booth and Snower, pp. 19-40. 